\title{
Editorial
}

\section{Falling Short: Continued Challenges in Meeting the Mental Health Needs of Children with Special Health Care Needs}

Nearly one in every five households has a child with a special health care need (CSHCN), and at least one quarter of these children also has a mental health problem. These data underscore the fact that $\mathrm{CSHCN}$ have substantial unmet mental health needs; for those with more chronic emotional or behavioral problems, unmet need is even higher (Inkelas et al. 2007). High levels of unmet need persist in the face of substantial resources that have been committed to improving care for these populations, including the integration of behavioral health into Medicaid managed care.

In this issue of $H S R$, Kuhlthau et al. further highlight the issue of unmet need in their examination of care for emotional health problems of CSHCN covered under Medicaid managed care and fee-for-service (FFS) plans. Although they identify more parent-reported unmet mental health care need for CSHCN in Medicaid managed care compared with FFS plans, the key story is not simply this difference, but the continued problem of the disparity between perceived mental health need and receipt of services. Moreover, Cooper's recent analysis of funding for children's mental health services raises questions about the misalignment between which services are covered and the appropriateness and quality of those that are provided, particularly for CSHCN (Cooper 2008). This mismatch could explain some of the continued unmet need.

Understanding this unmet need requires us to better determine the sources; to measure not only the commonly tracked processes of care, but also the mental health outcomes for CSHCN; to explore how mental health services can be better coordinated across the multiple systems that often serve these youth (e.g., schools, child welfare); and to identify opportunities for prevention by addressing other factors that contribute to poor mental health. Further, while CSHCN are a key part of the youth population needing mental health services, better understanding the nature of the care they 
receive-and its outcomes-can inform how best to serve other vulnerable youth populations, including those in foster care and the juvenile justice system.

In the sections that follow, we identify issues and action steps for researchers and policy makers seeking to better meet the mental health needs of CSHCN.

\section{Analyze what Constitutes Good Mental Health CARE MANAGEMENT FOR CSHCN}

CSHCN account for an estimated 76 percent of total Medicaid expenditures for children, even though they represent $<25$ percent of the population (Oehlmann 2004). The majority of CSHCN are eligible for Medicaid, and most CSHCN with mental health problems receive care paid for by Medicaid. In general, Medicaid managed care has improved efficiency, contained costs, and improved service coordination for children (Cooper and Kuhlthau 2001). For instance, in North Carolina, the introduction of a public sector managed Medicaid mental health care pilot resulted in reductions in child inpatient services and slower growth in mental health care costs (Burns et al. 1999). However, in that analysis, child mental health outcomes were not measured. An examination of Medicaid in the District of Columbia revealed that CSHCN in managed care had lower levels of unmet need, and their parents reported improved access, characterized by lower wait time and greater availability of specialist care (Mitchell and Gaskin 2007). Despite these encouraging findings, evidence regarding how youth with chronic emotional and behavioral problems are faring in Medicaid managed care models remain mixed at best.

To date, most analyses of how CSHCN are faring in Medicaid managed care rely on parent-reported indices of unmet mental health need. Kuhlthau et al. follow in that tradition, finding that a nationally representative sample of CSHCN with emotional or behavioral problems served by managed care plans actually had greater unmet mental health care need than those enrolled in FFS plans. Davidoff et al. (2006) found that parents of children with chronic mental health problems reported less mental health service use under managed care, especially when services are carved out. However, neither of these studies was able to definitively determine why some parents continued to report unmet mental health need. For example, it is difficult to know if this unmet mental health need is related to better care management (i.e., not 
needing as many services as parents expected), problems in mental health care access, or other factors. One reason is that the analyses focused on numbers of services received, rather than outcomes achieved. This is understandable, as the former are much easier to measure.

There is some early evidence to suggest that part of the problem for $\mathrm{CSHCN}$ in managed care may be due to a negative impact on the connections between mental health care and other care needed by youth with more serious emotional or behavioral problems. Stroul et al. (1998) analyzed data from the Health Care Reform Tracking Project (HCRTP) and found that managed care mental health services were not well-linked to other community services. They argue that, by contrast, before the advent of managed care, many communities made efforts to build a system of community-based care (e.g., day treatment, crisis services) involving multiple sectors (e.g., mental health, child welfare, education).

Understanding how mental health care is "managed" in managed care settings is critical. Although it is unlikely that there is a single care management model, there are likely vast differences between, for example, limiting the number of outpatient visits and careful coordination between outpatient specialty mental health providers and providers in school-based settings. Without a better understanding of the care management models and the outcomes that result, it is difficult to know just how to act on findings such as those reported here.

\section{Develop Outcomes-BASEd Evidence}

The studies described above, including that of Kuhlthau and colleagues, focus on parent perception of unmet mental health need. While this is consistent with the important principle of assessing the patient experience with care, it calls into question the origin of that unmet need. Much research has examined correlates of unmet mental health need (e.g., low SES, severity of disorder), but not enough attention has been paid to the explanations for it (Ganz and Tendulkar 2006). Current metrics do not allow us to tease potential explanations apart. Further complicating the picture, research tends to focus on parent perception of need, and rarely evaluates child perspectives, or for that matter, child functioning. According to the Maternal and Child Health Bureau, the core measures of adequately serving CSHCN include receipt of coordinated and ongoing care, "adequate" coverage for the breadth of services needed, and access to community-based service systems (McPherson 
et al. 2004). These core measures provide no information about a child's mental well-being or functioning, and do not necessarily reflect whether care is evidence-based. This is particularly troubling because ineffective care is often costly.

Some states, including Michigan, have begun to manage care by using validated assessment tools, such as the Child and Adolescent Functional Assessment Scale (Hodges \& Wong 1996; Cooper 2008). While this is promising, we do not yet know whether this approach to managing care will actually result in better outcomes. Further, there are other important outcomes to consider when assessing the effectiveness of mental health care. Because mental health problems can affect the trajectory of a person's life, care should be assessed with respect not only to cost savings but also to long-term outcomes (e.g., disability adjusted life years or DALYs). Early investments in mental health care can also have profound impact on child outcomes in other settings (e.g., school, employment, physical health) (Quinn and McDougal 1998). Given the complexity of needs for many CSHCN, these outcomes deserve attention.

\section{EXAMINE WAYS TO InTEGRATE MENTAL HEALTH SYSTEMS OF CARE FOR CSHCN}

The lack of integration among traditional mental health, education, child welfare, juvenile justice, and other youth service organizations is staggering. This results in children falling through the cracks, and duplicating or more often missing needed services (Thomas, Gourley, and Mele 2004). Missed opportunities to develop coordinated treatment plans across multiple systems that serve a child are frequent. This is not surprising, because protocols for assessing and tracking outcomes across several systems are generally lacking (VanDenBerg 1993).

Child mental health policymakers need to critically analyze the interfaces among the various agencies serving at risk youth, and to identify and remove structural and policy barriers in order to streamline service delivery. Mental health care provided through managed care should be better integrated with existing community services, increasing the use of available and appropriate community mental health care, not reducing access. This may lead to different financial arrangements between agencies, and a clearer understanding of where the return on investment in early intervention or mental health treatment may accrue. 


\section{EXPAND PREVENTION EFFORTS FOR MENTAL HeALth FOR CSHCN}

A key underlying issue, and one that receives far less attention, is how to address upstream factors that may contribute to children having such profound needs. For example, we need to understand more clearly the factors that produce a mentally healthy child and intervene early. Several studies on the effectiveness of early childhood education programs show a positive impact on socioemotional health, a benefit that could mitigate certain mental health problems before intensive intervention is necessary (Anderson et al. 2003). Health programs that boost social and emotional learning (SEL) competencies have great promise, but have not received adequate attention (Payton et al. 2000).

There are also opportunities to integrate prevention services into Medicaid managed care. While we need to test strategies that will effectively reduce the barriers to mental health service provision in Medicaid managed care for CSHCN, strategies will be more effective if they address the realities that have been hard to surmount (e.g., provider supply, lack of specialty care, limits on funding) with parallel efforts focused on primary and secondary prevention. Our best chance is to start to test models of mental health wellness in these managed care settings (e.g., tools for anxiety or stress reduction) and across agencies (e.g., working with schools) that may improve health outcomes and reduce costs. While federal funding in other areas of mental health and substance use have made concerted efforts to contribute dollars to prevention, this focus is not always present in Medicaid managed care. Now, more than ever, we need to assess the role prevention should play in a comprehensive behavioral health care plan, and how this would be covered by Medicaid.

Anita Chandra and Nicole Lurie

\section{REFERENCES}

Anderson, L. M., C. Shinn, M. T. Fulllilove, S. C. Scrimshaw, J. E. Fielding, J. Normand, V. G. Carnade-KulisTask Force on Community Preventive Services. 2003. "The Effectiveness of Early Childhood Development Programs: A Systematic Review." American Journal of Preventive Medicine 24 (3): 32-46. 
Burns, B. J., S. E. Teagle, M. Schwartz, A. Angold, and A. Holtzman. 1999. "Managed Behavioral Health Care: A Medicaid Carve-out for Youth." Health Affairs 18 (5): 214-25.

Cooper, J. L. 2008. "Towards Better Behavioral Health for Children, Youth and Families: Financing that Support Knowledge.” National Center for Children in Poverty.

Cooper, W. O., and K. Kuhlthau. 2001. "Evaluating Medicaid Managed Care Programs for Children." Ambulatory Pediatrics 1: 112-6.

Davidoff, A., I. Hill, B. Courtot, and E. Adams. 2007. "Effects of Managed Care on Service Use and Access for Publicly Insured Children with Chronic Health Conditions." Pediatrics 119 (5): 956-64.

Ganz, M. L., and S. Tendulkar. 2006. "Mental Health Care Services for Children With Special Health Care Need and Their Family Members: Prevalence and Correlates of Unmet Needs." Pediatrics 117 (6): 2138-48.

Hodges, K., and M. M. Wong. 1996. "Psychometric Characteristics of a Multidimensional Measure to Assess Impairment: The Child and Adolescent Functional Assessment Scale." Journal of Child and Family Studies 5: 445-67.

Inkelas, M., R. Raghavan, K. Larson, A. A. Kuo, and A. N. Ortega. 2007. "Unmet Mental Health Need and Access to Services for Children with Special Health Care Needs and Their Families." Ambulatory Pediatrics 7 (6): 431-8.

McPherson, M., G. Weissman, B. B. Strickland, P. C. Van Dyck, S. J. Blumberg, and P. W. Newacheck. 2004. "Implementing Community-Based Systems of Services for Children and Youths With Special Health Care Needs: How Well Are We Doing?" Pediatrics 113: 1538-44.

Mitchell, J., and D. Gaskin. 2007. "Caregivers' Ratings of Access: Do Children with Special Health Care Needs Fare Better Under Fee-for-Service or Partially Capitated Managed Care?" Medical Care 45 (2): 146-53.

Oehlmann, M. L. 2004. "Improving Managed Care for Children with Special Needs: A Best Clinical and Administrative." Practices Toolkit. Princeton, NJ: Center for Health Care Strategies.

Payton, J. W., D. M. Wardlaw, P. A. Graczyk, M. R. Bloodworth, C. J. Tompsett, and R. P. Weissberg. 2000. "Social and Emotional Learning: A FrameWork for Promoting Mental Health Reducing Risk Behaviors in Children and Youth.” Journal of School Health 70 (5): 179-85.

Quinn, K. P., and J. L. McDougal. 1998. "A Mile Wide and a Mile Deep: Comprehensive Interventions for Children and Youth with Emotional and Behavioral Disorders and their Families." School Psychology Review 27 (2): 191-203.

Saunders, R. C., and C. A. Heflinger. 2003. "Access to and Patterns of Use of Behavioral Health Services among Children and Adolescents in TennCare." Psychiatric Services 54: 1364-71.

Stroul, B. A., S. A. Pires, M. I. Armstrong, and J. C. Meyers. 1998. "The Impact of Managed Care on Mental Health Services for Children and Their Families." The Future of Children 8 (2): 119-34. 
Tang, M. H., K. S. Hill, A. A. Boudreau, R. M. Yucel, J. M. Perrin, and K. A. Kuhlthau. 2008. "Medicaid Managed Care and the Unmet Need for Mental Health Care among Children with Special Health Care Needs." Health Services Research.

Thomas, J., G. K. Gourley, and N. Mele. 2004. "The Impact of Managed Behavioral Health Care on Youth in the Juvenile Justice System." Archives of Psychiatric Nursing 18 (4): 135-42.

VanDenBerg, J. E. 1993. "Integration of Individualized Mental Health Services into the System of Care for Children and Adolescents." Administration and Policy in Mental Health and Mental Health Services Research 20 (4): 247-5. 